\title{
Norepinephrine transporter promotes the invasion of human colon cancer cells
}

\author{
HUAHUA ZHANG ${ }^{1,2}$, JIYU MIAO ${ }^{2}$, FANG LI $^{2}$, WANJUAN XUE ${ }^{1,2}$, KAIJIE TANG $^{2}$, XIAOGE ZHAO $^{3}$, \\ XINTAO JING ${ }^{2}$, JING ZHANG ${ }^{1}$, CHEN HUANG ${ }^{2,3}$, NI HOU ${ }^{2}$ and JIMING HAN ${ }^{1}$ \\ ${ }^{1}$ Medical Research and Experimental Center, Medical College, Yan'an University, Yan'an, Shaanxi 716000; \\ ${ }^{2}$ Department of Cell Biology and Genetics, School of Basic Medical Sciences; ${ }^{3}$ Key Laboratory of Environment and \\ Genes Related to Diseases, Xi'an Jiaotong University Health Science Center, Xi'an, Shaanxi 710061, P.R. China
}

Received November 26, 2018; Accepted August 29, 2019

DOI: $10.3892 / 01.2019 .11146$

\begin{abstract}
Epidemiological studies suggested the use of antidepressants to be associated with decreased risk of colorectal cancer (CRC). However, the underlying mechanism through which this decreased risk occurs remains elusive. The norepinephrine transporter (NET) is a target of antidepressants that maintains noradrenergic transmission homeostasis; however, little is known about its function in human CRC cells. The present study, using public datasets and immunohistochemistry approaches, revealed that NET was highly expressed in human CRC tissues with metastasis and in human colon cancer cells. Furthermore, knockdown of NET inhibited the invasive capability of human colon cancer cells. Additionally, epithelial (E)-cadherin expression was increased and Notch1 signaling was inhibited in NET-depleted colon cancer cells. These findings suggest that NET is highly expressed in human colon cancer, which is associated with the invasion of human colon cancer cells by influencing cell-cell adhesion through the Notch1-E-cadherin pathway. Thus, the present study revealed a novel function for NET and its downstream effectors in colon cancer cells, which will be valuable for future studies in a clinical setting.
\end{abstract}

\section{Introduction}

Colorectal cancer (CRC) is the third most common malignancy worldwide in 2012 (1). The incidence, in developing countries,

Correspondence to: Professor Ni Hou, Department of Cell Biology and Genetics, School of Basic Medical Sciences, Xi'an Jiaotong University Health Science Center, 76 Western Yanta Road, Xi'an, Shaanxi 710061, P.R. China

E-mail: houni@xjtu.edu.cn

Professor Jiming Han, Medical Research and Experimental Center, Medical College, Yan'an University, 38 Guanghua Road, Yan'an, Shaanxi 716000, P.R. China

E-mail:yadxhjm@163.com

Key words: norepinephrine transporter, invasion, human colon cancer cell has increased by 2 -4-fold over the last two decades $(2,3)$. Similar to other malignancies, metastases are the primary cause of CRC-associated mortality (4). The discovery of new therapeutic strategies or potential co-therapeutic agents against CRC will be of benefit to numerous patients. Patients with this malignancy often experience depression due to various stresses, which can aggravate clinical manifestations and, as a result, affect disease progression, prognosis and outcome $(5,6)$. The inhibition of monoamine transmitter reuptake by targeting monoamine transporters is currently the most successful mechanism for treating depression (7). Epidemiological and animal studies suggest that the use of antidepressants may be associated with decreased risk of CRC (8-10). However, the mechanism underlying this decreased risk remains elusive.

The norepinephrine transporter (NET) belongs to the monoamine transporter class; monoamine transporters are located in the neurons and glial cells of the central nervous system and in tissues of the peripheral organs innervated by the sympathetic ganglia, including the heart, adrenal medulla, gastrointestinal tract and placenta (11). Upon binding to its substrate, norepinephrine (NE), NET co-transports NE along with a $\mathrm{Na}^{+}$ion into the cytoplasm to maintain noradrenergic transmission homeostasis, which is targeted in the treatment of depression $(12,13)$. NET not only regulates the longevity of $\mathrm{NE}$ in the synapse but also plays a role in presynaptic and postsynaptic homeostasis. Several intracellular and extracellular signaling molecules can regulate its function (12-14). In addition, loss or disruption of NET function, caused by factors such as genetic polymorphisms, has been epidemiologically demonstrated to be closely associated with various neuropsychiatric diseases, cardiovascular diseases and cancer (15-17).

The loss of cell adhesion is a key step in the cascade leading to malignancy and metastasis. In numerous instances, epithelial tumors lose epithelial (E)-cadherin-mediated adhesions as they progress toward malignancy (18). Preoperatively elevated soluble E-cadherin levels are a prediction marker of metastasis and a pre-therapeutic prognostic marker for patients with CRC and hepatic metastases $(19,20)$. Notch signaling, a critical pathway in tissue development, was also reported to contribute to tumorigenesis and tumor metastasis (21). Furthermore, Notch signaling was demonstrated to regulate E-cadherin expression in breast cancer, hepatocarcinoma and 
CRC (22-24). The loss of NET function was found to be associated with genetic alterations in both neural crest cells and the adult superior cervical ganglion and locus ceruleus; the inhibitor of Notch signaling numb-like (Numbl) exhibited increased expression and the Notch signaling pathway was subsequently inhibited in NET-deficient mice (25). However, little is known about the function of NET and its downstream mediators in cancer.

In the present study, the significance of NET in CRC was determined using publicly available CRC gene expression RNA sequencing (RNAseq) datasets from The Cancer Genome Atlas (TCGA) database and by immunohistochemistry assays. NET was highly expressed and associated with CRC metastasis. Subsequently, the effect of NET depletion was determined by transfection of human colon cancer HCT116 and SW480 cells with NET-targeting small interfering (si)RNA. Knockdown of NET resulted in decreased invasion of CRC cells, inhibition of Notch1 activation and increased E-cadherin expression. These findings revealed that NET was highly expressed and closely associated with the invasion of human CRC cells by influencing cell-cell adhesion through the Notch1-E-cadherin pathway.

\section{Materials and methods}

Materials. Antibodies against human NET (cat nos. GTX47102 and Ab41559) were purchased from GeneTex, Inc. and Abcam, respectively. Anti-E-cadherin (cat. no. 20874-1-AP), anti-N-cadherin (cat. no. 22018-1-AP), anti-Notch1 (cat. no. 20687-1-AP) and anti-Snail1 (cat. no. 13099-1-AP) were purchased from Wuhan Sanying Biotechnology, while anti-GAPDH (cat. co. Sc-32233) was obtained from Santa Cruz Biotechnology, Inc.

Bioinformatics analysis. Data of NET expression in tumor tissues and Tumor-Node-Metastasis (TNM) classification of 183 CRC cases were extracted from the RNAseq Illumina HiSeq dataset in TCGA of the University of California Santa Cruz Genomics Institute. The datasets were downloaded from TCGA tools cancer browser (https://xenabrowser.net/hub/). As the previous reports $(26,27)$ demonstrated, the procedure of select datasets (TCGA.COADREAD.sampleMap/HiSeqV2).

Clinical tissue specimens. Formalin-fixed paraffin-embedded tissue blocks of CRC tumors and adjacent normal mucosal tissues were obtained from 35 patients between December 2017 and June 2018 at the Second Affiliated Hospital of Xi'an Jiaotong University (Xi'an, China). None of the patients received prior chemotherapy, radiotherapy or systemic therapies, or had additional malignant tumors. The clinicopathological parameters of the patients are presented in Table SI. There were 15 cases with metastasis, including lymph node metastasis and distant metastasis, and 20 cases without metastasis. All samples were obtained with the informed consent of each patient before collection. The present study was approved by the Medical Ethics Committee of Xi'an Jiaotong University (approval no. 2017-488).

Tissue immunohistochemistry. Immunohistochemical detection of NET was performed as previously described (28).
Anti-NET antibody was used at 1:100 dilution and incubated overnight at $4^{\circ} \mathrm{C}$; the human Biotin-Streptavidin HRP Detection kit (cat. no. SP-9001; ZSGB-BIO) including the anti-rabbit secondary antibody (incubation for $30 \mathrm{~min}$ at room temperature) and DAB chromogenic agents (incubation for $15 \mathrm{~min}$ at room temperature) were used according to the manufacturer's protocol. All samples were counterstained with hematoxylin for $30 \mathrm{sec}$ at room temperature.

NET positivity appeared as brown or yellow staining in the plasma membrane or cytoplasm. Scores by two independent investigators were averaged for evaluation of NET expression. The proportion of positive cells was scored as follows: 0 , no positive cells; $1,<10 \%$ positive cells; $2,10-50 \%$ positive cells; $3,50-80 \%$ positive cells; and $4, \geq 80 \%$ positive cells. Staining intensity was rated as follows: 0 , no staining; 1 , light yellow and weak staining; 2, yellow and moderate staining; and 3 , brown and strong staining. The product of staining intensity and proportion of positive cells comprised the staining index (SI). The ratio of NET SI of CRC tumor tissues to NET SI of adjacent normal mucosal tissues was used to evaluate differences in NET expression between patients with metastatic and non-metastatic CRC.

Cell lines and cell culture. Human colorectal cancer cells (HCT116, RKO, HT29, SW480, SW620 and CaCo-2), purchased from the American Type Culture Collection (ATCC), were kindly provided by Professor Hiroyuki Kuwano (Graduate School of Medicine, Gunma University, Maebashi, Japan). The normal colonic epithelial cell line NCM460 was purchased from the ATCC. The cells were cultured in RPMI-1640 medium (Hyclone; GE Healthcare Sciences) supplemented with $10 \%$ fetal bovine serum (FBS; Biological Industries) at $37^{\circ} \mathrm{C}$ in a humidified atmosphere of $95 \%$ air and $5 \% \mathrm{CO}_{2}$, with medium change every 2 days. Cells in the mid-log phase were used for the experiments in the present study.

siRNA synthesis and transfection. Human NET siRNAs (siNET1 sense, 5'-GAUUUCGUGACUGUAGUUU-3'; siNET1 antisense, 5'-AAACUACAGUCACGAAAUC-3'; siNET2 sense, 5'-GGAGAAGGAGAGCUACCAA-3'; and siNET2 antisense, 5'-UUGGUAGCUCUCCUUCUCC-3') and negative control siRNA (siNC sense, 5'-UUCUCCGAACGU GUCACGU-3'; and siNC antisense, 5'-ACGUGACACGUU CGGAGAA-3') were chemically synthesized by Shanghai Jima Industrial, Co., Ltd. Lipofectamine ${ }^{\circledR} 2000$ (Invitrogen; Thermo Fisher Scientific, Inc.) was used to transfect the cells. The siRNAs were diluted to $30 \mathrm{nM}$ in the wells plated with HCT116 or SW480 cells. Following incubation for $12 \mathrm{~h}$, cells were incubated with normal medium to observe changes in cell viability or harvested to be seeded into a 24-well cell culture Transwell insert system ( $8 \mu \mathrm{m}$ pore size; Becton Dickinson and Company) for observing changes in cell invasion. The transfection efficiency was determined using reverse transcription-quantitative (RT-q)PCR and western blot analysis.

$R N A$ isolation and RT-qPCR. Total cellular RNA from the human colorectal cancer cells was extracted using TRIzol ${ }^{\circledR}$ reagent (Invitrogen; Thermo Fisher Scientific, Inc.) according to the manufacturer's instructions. mRNA was reverse 
A

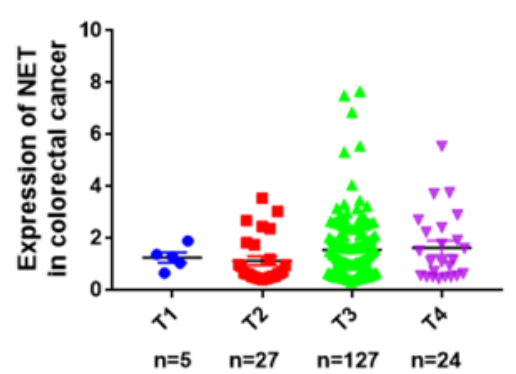

C

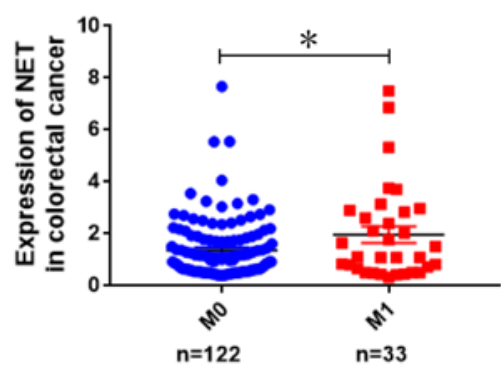

B

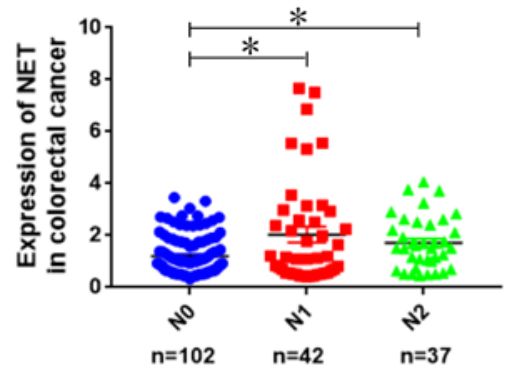

D

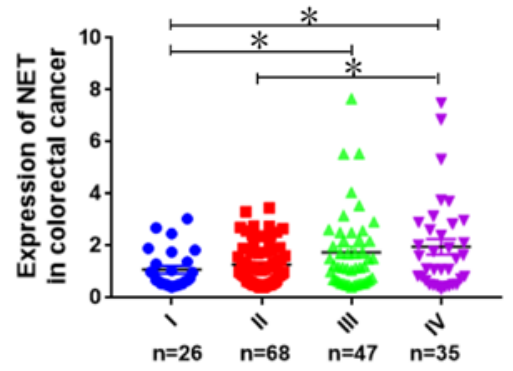

Figure 1. Analysis of NET expression in human CRC using TCGA database. Using publicly available CRC gene expression RNAseq datasets in TCGA database, the expression patterns of NET were analyzed bioinformatically and shown according to the grade of (A) topography (T1, T2, T3 and T4), (B) lymph node metastasis (N0, N1 and N2), (C) distant metastasis (M0 and M1) and (D) clinical stages (I, II, III and IV) of patients with CRC. Kruskal-Wallis test followed by least significance difference post hoc test was used to analyze NET expression among different T, N, M and clinical stage groups. ${ }^{*} \mathrm{P}<0.05$. TCGA, The Cancer Genome Atlas; NET, norepinephrine transporter; CRC, colorectal cancer.

transcribed into complementary DNA (cDNA) (at $37^{\circ} \mathrm{C}$ for $30 \mathrm{~min}$ and $85^{\circ} \mathrm{C}$ for $5 \mathrm{~min}$ ) using the PrimeScript RT Reagent kit (Takara Bio, Inc.). qPCR was performed using the FastStart Essential DNA Green Master kit (Roche Diagnostics) on a LightCycler ${ }^{\circledR} 480$ II instrument, according to the manufacturer's instructions: $95^{\circ} \mathrm{C}$ for $10 \mathrm{~min} ; 95^{\circ} \mathrm{C}$ for $15 \mathrm{sec}, 60^{\circ} \mathrm{C}$ for $1 \mathrm{~min}, 72^{\circ} \mathrm{C}$ for $30 \mathrm{sec}\left(40\right.$ cycles); and $94^{\circ} \mathrm{C}$ for $90 \mathrm{sec}$, $60^{\circ} \mathrm{C}$ for $3 \mathrm{~min}$ and $94^{\circ} \mathrm{C}$ for $10 \mathrm{sec}$. The relative expression of NET to $\beta$-actin (internal control) was calculated using the $2^{-\Delta \Delta \mathrm{Cq}}$ method (29). The primers were synthesized by Tsingke Biotech, and the sequences were as follows: NET forward, 5'-GCGCTCATCCCAGTGTCTAA-3'; NET reverse, 5'-GGA TCAAGAAGGCACCGCC-3'; $\beta$-actin forward, 5'-CCAGAG GCGTACAGGGATAG-3'; and $\beta$-actin reverse, 5'-CCAACC GCGAGAAGATGA-3'. All reactions were performed in triplicate.

Cell invasion assay. Cell invasion was examined in a 24-well cell culture Transwell insert system. The chambers were coated with Matrigel for determination of cell invasive ability. The lower chamber was filled with $600 \mu 1$ RPMI-1640 medium containing $10 \% \mathrm{FBS}$, and the upper chamber was filled with $200 \mu$ RPMI-1640 medium without FBS. HCT116 $\left(6 \times 10^{4}\right)$ and SW480 $\left(10 \times 10^{4}\right)$ cells were seeded onto the upper chamber. Following incubation for $24 \mathrm{~h}$, the remaining cells in the upper chamber were completely removed using a cotton swab, and the invading cells on the bottom surface were fixed with $4 \%$ polyformaldehyde for $15 \mathrm{~min}$ at room temperature and stained with $0.1 \%$ crystal violet for $30 \mathrm{~min}$ at room temperature. Digital images were obtained using a photomicroscope at x100 magnification (Nikon Corporation). In addition, stained cells on the lower surface of the chamber were dissolved with $150 \mu \mathrm{l}$ DMSO and quantified by measuring the absorbance at $590 \mathrm{~nm}$ using a microplate reader (BMG Labtech $\mathrm{GmbH})$.

Cell viability assay. Cells (HCT116, 3×10 3 cells/well; and SW480, $3.5 \times 10^{3}$ cells/well) were seeded onto a 96 -well plate. Following incubation for $24 \mathrm{~h}$, MTT $(0.5 \mathrm{mg} / \mathrm{ml})$ was added and incubated at $37^{\circ} \mathrm{C}$ for $4 \mathrm{~h}$, and the absorbance of each well was determined spectrophotometrically at $570 \mathrm{~nm}$ using the FLUOstar OPTIMA (BMG Labtech GmbH). All samples were evaluated in quintuplicate.

Western blot analysis. Cells were harvested from culture dishes and lysed in RIPA buffer supplemented with protease inhibitors and phosphatase inhibitors (Invitrogen; Thermo Fisher Scientific, Inc.). The protein concentration was determined using the Pierce BCA Protein Assay Kit (Thermo Fisher Scientific, Inc.). The cell lysates (20 $\mu \mathrm{g} /$ lane) were separated by SDS-PAGE on a $10 \%$ polyacrylamide gel and transferred to PVDF membranes for blotting. The membranes were blocked in 5\% skim milk for $30 \mathrm{~min}$ at room temperature and incubated with primary antibodies (all $1: 1,000)$ at $4^{\circ} \mathrm{C}$ overnight. An antibody against GAPDH was used as the loading control. This was followed by incubation with horseradish peroxidase-conjugated IgG anti-rabbit (cat. no. 111-035-144) and anti-mouse (cat. no. 115-035-146) secondary antibodies (Jackson ImmunoResearch Laboratories, Inc.) at 1:5,000 dilution for $1 \mathrm{~h}$. The immunoreactive bands were visualized by enhanced chemiluminescence (EMD Millipore). The band 
A

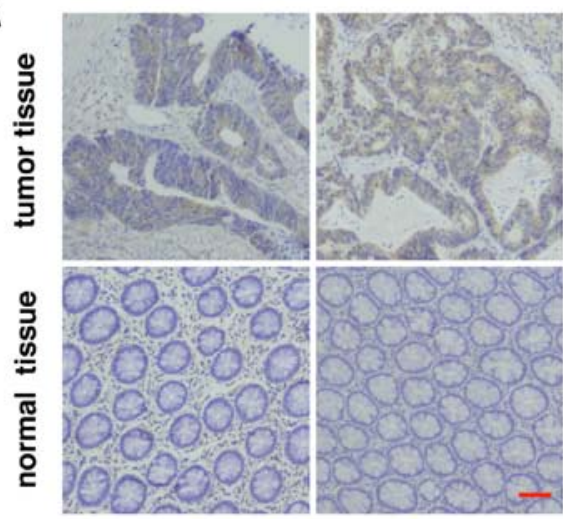

without metastasis with metastasis

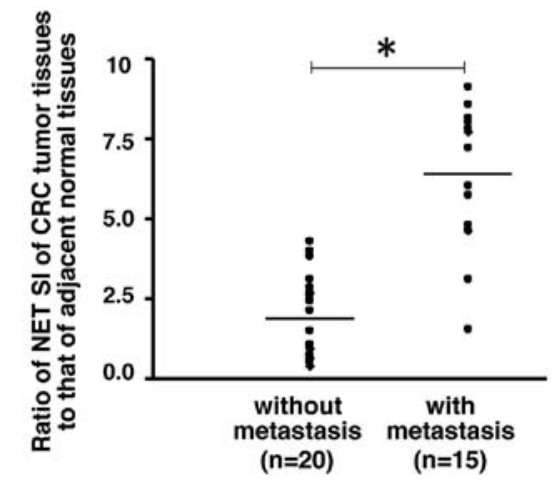

B
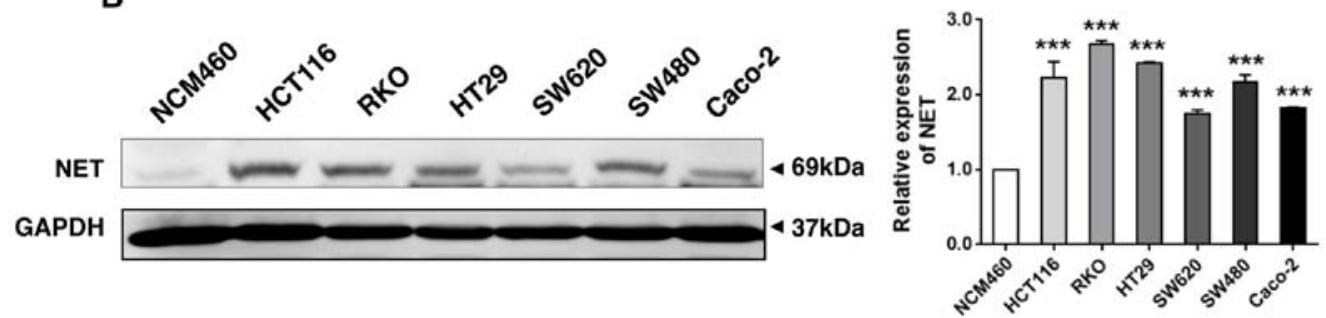

Figure 2. NET is highly expressed in human CRC with metastasis and in human CRC cells. (A) Clinical CRC tissues and adjacent normal tissues were collected and used for paraffin sections. NET immunohistochemistry was performed, which revealed increased expression of NET in cancer tissues of patients with metastatic CRC (scale bar, $50 \mu \mathrm{m}$ ). The proportion and intensity of staining were evaluated and used to calculate the staining index (SI). The ratio of NET SI of CRC tumor tissues to NET SI of adjacent normal mucosal tissues was used to evaluate differences in NET expression between patients with metastatic and non-metastatic CRC. One-way ANOVA followed by Tamhane's T2 post hoc test was used to compare the expression of NET between tumor tissues of patients with CRC with metastasis and that of those without metastasis. (B) Lysates of human CRC cell lines (HCT116, RKO, HT29, SW620, SW480 and CaCo-2) and a normal colonic epithelial cell line, NCM460, were harvested. Western blotting revealed high expression of NET in human CRC cells (left). The band intensities were quantified, and the relative expression of NET to GAPDH was calculated and normalized to the NCM460 cell line. One-way ANOVA and least significance differenceposthoc test were used to conduct multiple comparisons between the NET expression levels in human CRC cell lines and in NCM460 cells. ${ }^{*} \mathrm{P}<0.05,{ }^{* * *} \mathrm{P}<0.001$. CRC, colorectal cancer; NET, norepinephrine transporter.

intensity was measured by densitometry and quantified using the Gel Plotting Macros of NIH Image 1.62 software (National Institutes of Health). The relative expression ratio of NET to GAPDH was calculated and normalized to the siNC samples on the same membrane.

Statistical analysis. All experiments were performed with three independent replicates. The data were expressed as the mean \pm standard deviation and analyzed with SPSS 17.0 software (SPSS, Inc.). The Kruskal-Wallis and least significance difference (LSD) post hoc test were used to analyze the expression of NET among patients with CRC exhibiting different T, N and M. One-way ANOVA followed by Tamhane's T2 post hoc test was used to determine the associations between NET expression level and clinical characteristics of patients with $\mathrm{CRC}$. $\mathrm{P}<0.05$ was considered to indicate a statistically significant difference.

\section{Results}

NET is highly expressed in CRC tissues with metastasis and in human colon cancer cells. In order to determine the significance of NET in CRC, publicly available CRC gene expression RNAseq datasets from TCGA database were analyzed. NET expression was not associated with tumor topography (Fig. 1A); however, it was significantly associated with lymphatic metastasis ( $\mathrm{P}<0.05$; Fig. 1B), distant metastasis $(\mathrm{P}<0.05$; Fig. 1C) and

Table I. Sequences of primers and oligonucleotides used in the present study.

Name $\quad$ Sequence (5' to 3')

siNET1 sense

siNET1 antisense

siNET2 sense

siNET2 antisense

siNC sense

siNC antisense

NET forward

NET reverse

$\beta$-actin forward

$\beta$-actin reverse

NET, norepinephrine transporter; NC, negative control; si, small interfering.

clinical stages $(\mathrm{P}<0.05$; Fig. 1D) in patients with $\mathrm{CRC}$ (30). The expression of NET was further examined in $35 \mathrm{CRC}$ tissues and adjacent normal tissues (Fig. 2A). In tumor tissues of patients with metastatic CRC, the expression of NET was significantly higher than in adjacent normal tissues, and its fold increase 
A

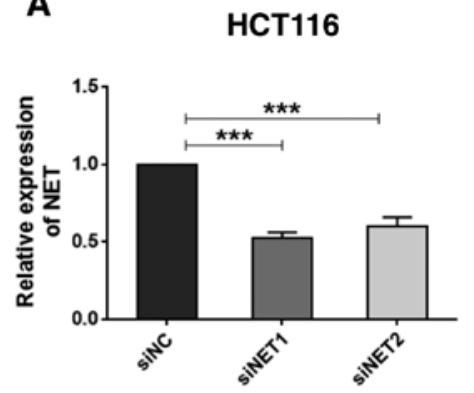

B
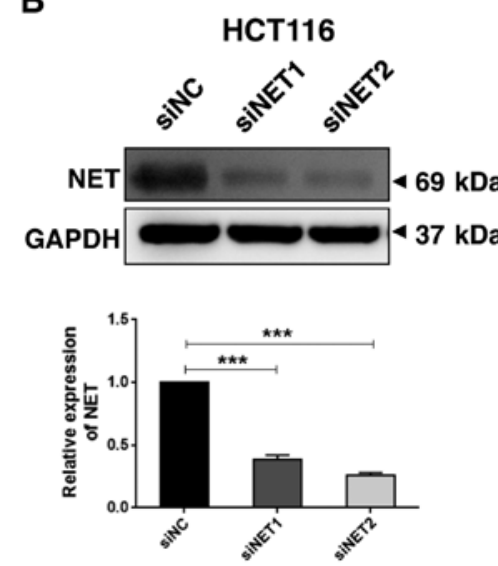

SW480

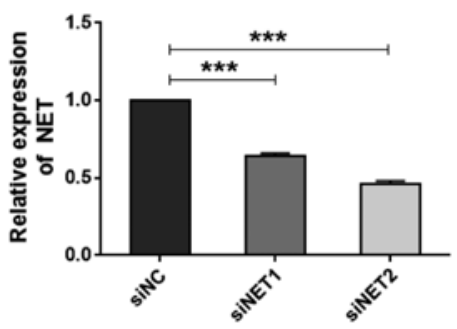

SW480
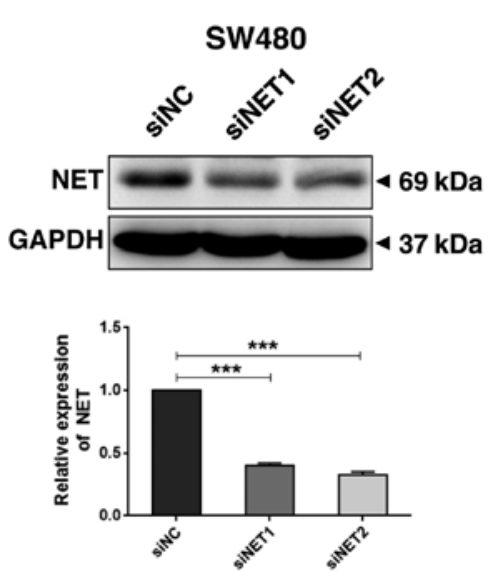

Figure 3. NET expression is decreased by siNET transfection in human colon cancer cells. HCT116 and SW480 cells were treated with NET-targeting siRNAs (siNET1 and siNET2) or negative control siRNA (siNC). (A) Reverse transcription-qPCR and (B) western blotting were performed and revealed a significant decrease in the mRNA and protein levels of NET in siNET1- and siNET2-transfected human colorectal cancer cells, compared with those observed in siNC-transfected cells. The band intensities of NET were quantified relative to GAPDH and normalized to the siNC sample. The experiments were repeated three independent times with reproducible results. One-way ANOVA and least significance difference post hoc test were used to conduct multiple comparisons between the expression levels of NET in siNET- and siNC-transfected cells. ${ }^{* * *} \mathrm{P}<0.001$. NET, norepinephrine transporter; siRNA, small interfering RNA.

was higher than that of patients with non-metastatic CRC. The protein levels of NET in CRC HCT116, RKO, HT29, SW480, SW620 and CaCo-2 cells were significantly higher than those in normal colonic epithelial NCM460 cells ( $\mathrm{P}<0.001$; Fig. 2B). These findings indicate that NET is highly expressed in CRC and is associated with CRC metastasis.

Knockdown of NET inhibits the invasive capability of human colon cancer cells. In order to determine the role of high NET expression in CRC metastasis, two siRNAs specifically targeting human NET (siNET1 and siNET2) and a negative control (siNC) were synthesized (Table I). Due to the high expression level of NET in human colon cancer HCT116 and SW480 cells (Fig. 2B), these cells were selected for use in the subsequent experiments. HCT116 and SW480 cells were transfected with siNET1, siNET2 or siNC, and total RNA and protein lysates were extracted at $48 \mathrm{~h}$ post-transfection. RT-qPCR and western blot analysis revealed significantly decreased mRNA $(\mathrm{P}<0.001$; Fig. 3A) and protein $(\mathrm{P}<0.001$; Fig. 3B) expression levels of NET in HCT116 and SW480 cells transfected with both siNET1 and siNET2, compared with those observed in cells transfected with siNC. Subsequently, the effect of NET knockdown on cell invasion was examined. As shown in Fig. 4, the Transwell assay demonstrated significantly suppressed invasive capability of siNET1- and siNET2-transfected HCT116 and SW480 cells compared with that of cells transfected with siNC; with decreases of 38.1 and $46.0 \%$ observed for HCT116 cells, and 42.3 and $49.0 \%$ observed for SW480 cells, respectively. In addition, there were no significant differences in cell viability between the siNET and siNC groups (Fig. S1). These findings indicate that the depletion of NET inhibits the invasive capabilities of human colon cancer cells.

Knockdown of NET increases E-cadherin and inhibits Notch1 signaling activity. E-cadherin is an important cell-cell adhesion molecule. Several studies indicated that expression of E-cadherin was negatively correlated with the degree of differentiation, invasiveness and metastasis of malignant tumors, and positively correlated with prognosis (18-20). Thus, the changes in the expression of E-cadherin were determined following the knockdown of NET in HCT116 and SW480 cells. As shown in Fig. 5, although there were no significant changes in $\mathrm{N}$-cadherin protein level, the protein level of E-cadherin was significantly increased in HCT116 and SW480 cells transfected with siNET1 $(\mathrm{P}<0.01$ and $\mathrm{P}<0.05$, respectively) and siNET2 $(\mathrm{P}<0.001$ and $\mathrm{P}<0.05$, respectively) compared with that observed in cells transfected with siNC. Moreover, compared with those of the siNC group, the expression levels of full length Notch1, cleaved Notch1 and the downstream transcription factor Snaill (31) were all significantly decreased in both HCT116 and SW480 cells transfected with siNET1 or siNET2, indicating Notch1 signaling inhibition in NET-depleted human CRC cells (Fig. 6). In addition to the key roles during the normal development of multiple tissues and organs, Notch signaling was shown to be associated with the occurrence and development of invasion and metastasis 


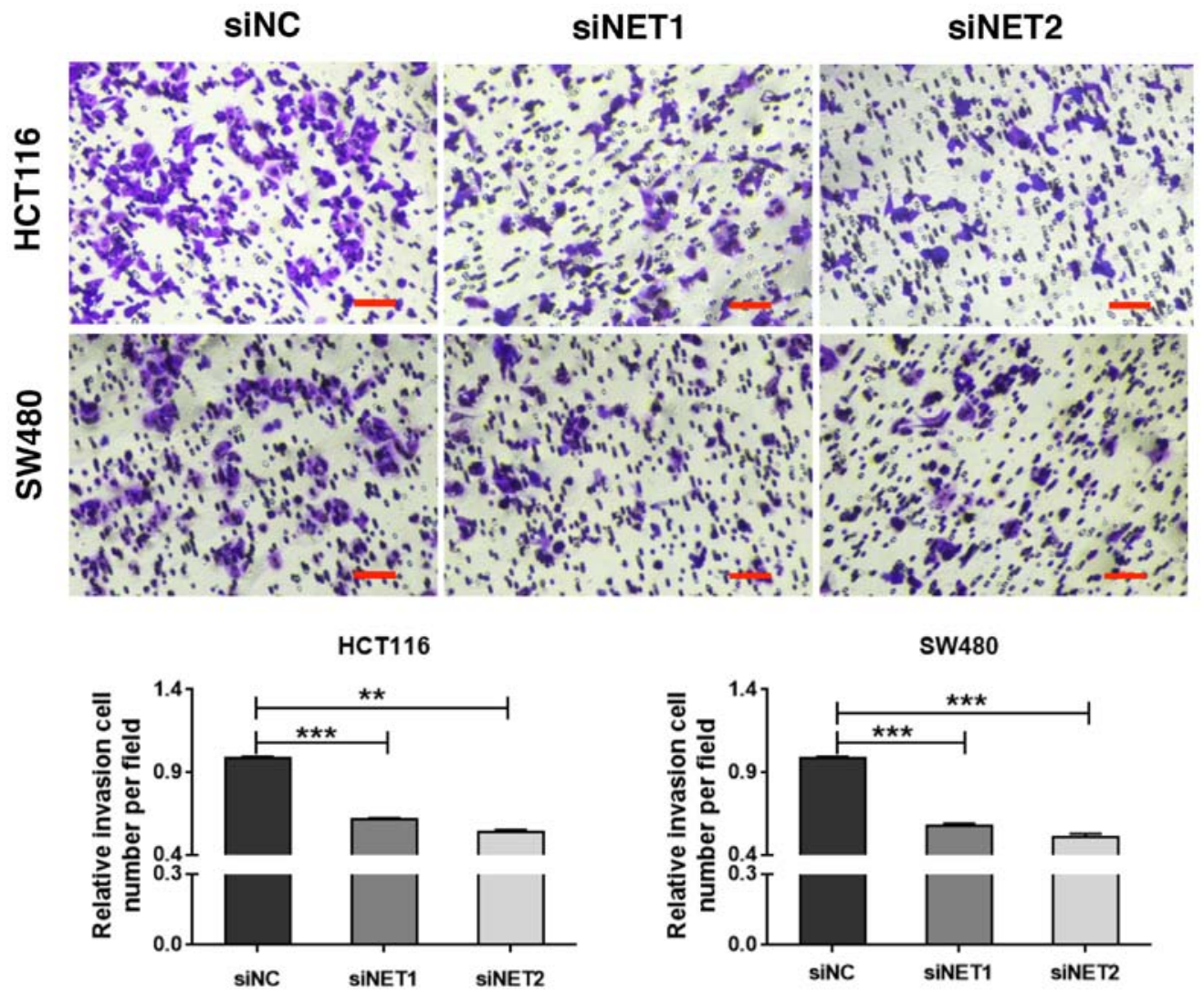

Figure 4. Knockdown of NET inhibits the invasive capability of human colon cancer cells. HCT116 and SW480 cells were treated with NET-targeting siRNAs (siNET1 and siNET2) or negative control siRNA (siNC). Transwell assay demonstrated a significant decrease in the number of invasive cells in siNET1- and siNET2-transfected human colorectal cancer cells compared with that observed in siNC-transfected cells. Scale bar, $100 \mu \mathrm{m}$. The experiments were repeated three independent times with reproducible results. One-way ANOVA was used to compare data between siNET- and siNC-transfected cells. Least significance difference tests were used as posthoc tests to conduct multiple comparisons. ${ }^{* *} \mathrm{P}<0.01,{ }^{* * *} \mathrm{P}<0.001$. NET, norepinephrine transporter; siRNA, small interfering RNA.
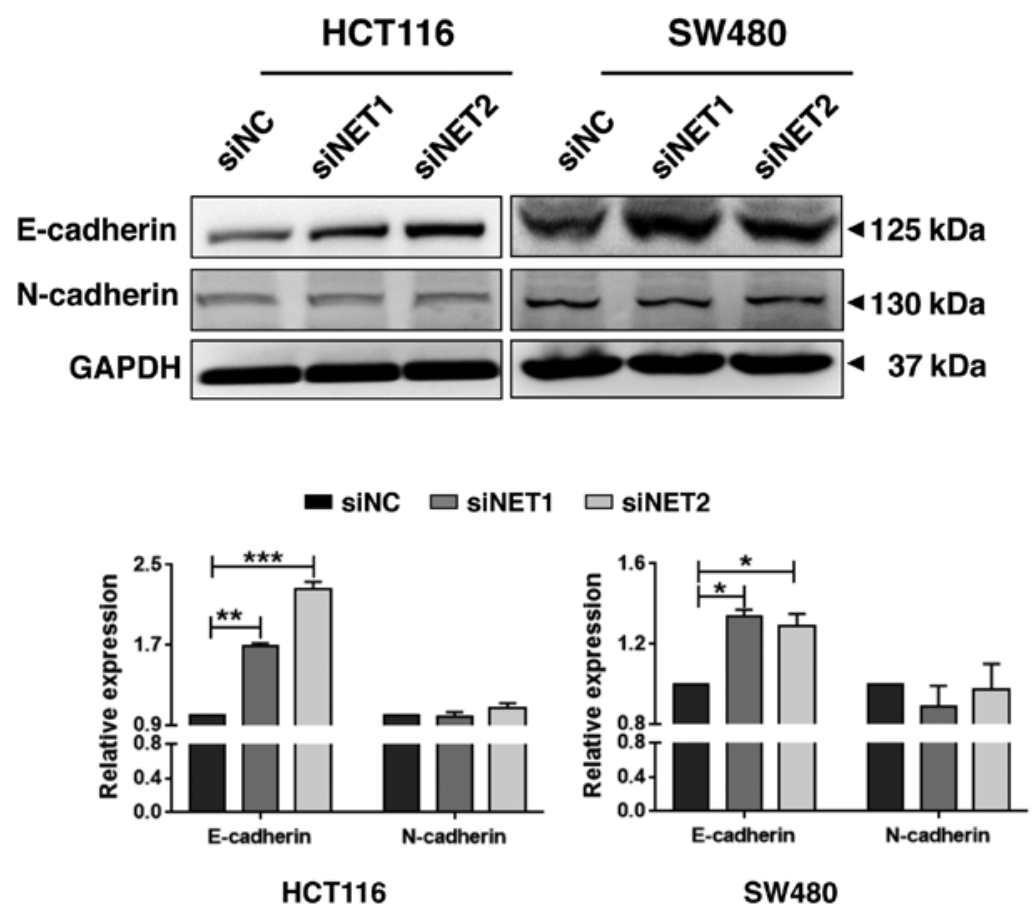

Figure 5. Knockdown of NET increases E-cadherin levels in human colon cancer cells. HCT116 and SW480 cells were treated with NET-targeting siRNAs (siNET1 and siNET2) or negative control siRNA (siNC). After $48 \mathrm{~h}$, cell lysates were harvested, and the protein samples were separated by SDS-PAGE. The levels of E-cadherin and N-cadherin were detected using western blotting. GAPDH was used as the loading control. The relative band intensities of NET vs. GAPDH were quantified and normalized to the siNC samples. The data are representative of three independent experiments. One-way ANOVA was used to compare the data between siNET- and siNC-transfected cells. The least significance difference test was used as the post hoc test to conduct multiple comparisons. ${ }^{*} \mathrm{P}<0.05,{ }^{* *} \mathrm{P}<0.01,{ }^{* * *} \mathrm{P}<0.001$. NET, norepinephrine transporter; siRNA, small interfering RNA. 

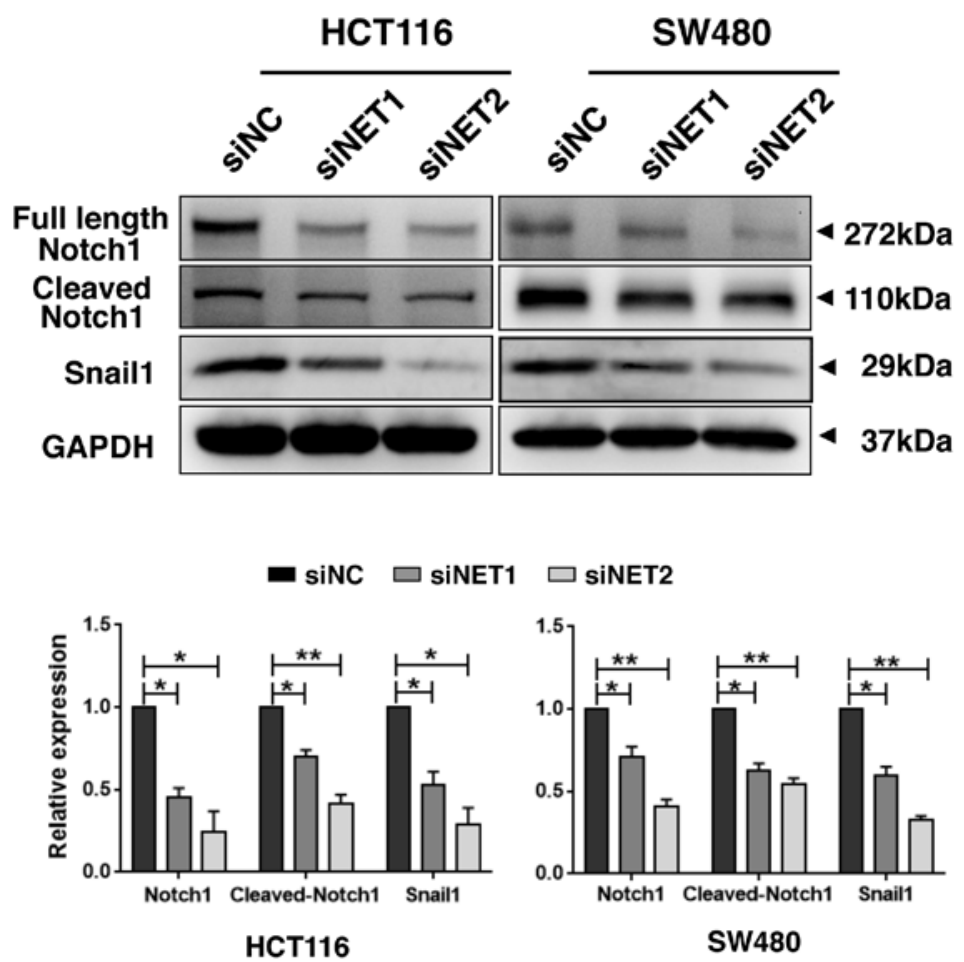

Figure 6. Depletion of NET inhibits Notch1 signaling in human colon cancer cells. HCT116 and SW480 cells were treated with NET-targeting siRNAs (siNET1 and siNET2) or negative control siRNA (siNC). After $48 \mathrm{~h}$, cell lysates were harvested, and the protein samples were separated by SDS-PAGE. The levels of full length Notch1, cleaved Notch1 and Snaill were detected by western blotting, and GAPDH was used as the loading control. The band intensities of NET relative to GAPDH were quantified and normalized to the siNC sample. The data are representative of three independent experiments. One-way ANOVA was used to compare data between siNET- and siNC-transfected cells. The least significance difference test was used as the post hoc test to conduct multiple comparisons. ${ }^{*} \mathrm{P}<0.05,{ }^{* *} \mathrm{P}<0.01$. NET, norepinephrine transporter; siRNA, small interfering RNA.

in various types of cancer. Chen et al (22) revealed that hypoxia-mediated Notch signaling may have an important role in the initiation of epithelial-mesenchymal transition and possess subsequent potential for breast cancer metastasis. Wang et al (23) demonstrated that abnormal Notch1 expression is strongly associated with metastatic hepatocellular carcinoma, which may be mediated through the Notch1-Snail 1-E-cadherin signaling pathway. Vinson et al summarized that Notch1 signaling regulates the formation and maintenance of colorectal cancer stem cells, which lead to metastasis and tumorigenesis $(21-23,31)$. Furthermore, Notch signaling was demonstrated to regulate E-cadherin expression in several types of cancer, including in CRC cells, and Notch1-Hairy enhancer of Split-1 (HES1)-E-cadherin was shown to promote invasiveness and metastasis, and was associated with poor survival (24). Combined with the findings of the present study, it is speculated that the depletion of NET results in the inhibition of Notch1 signaling, increases E-cadherin expression and decreases the invasive capability of human colon cancer cells.

\section{Discussion}

Epidemiological and in vivo studies suggested that the use of antidepressants was correlated with decreased risk of CRC (8-10). However, the mechanism underlying this decreased risk remains elusive. NET, a target of antidepressants, is distributed within neurons, glial cells and peripheral sympathetic nerve fibers that innervate tissue organs, including the gastrointestinal tract. The loss or disruption of NET function was shown to be associated with several neuropsychiatric diseases and tumors, for which the underlying mechanisms are unknown. Studies focusing on the SNP 1287 G/A (rs5569), located in exon 10 of hNET, have demonstrated an association with depression, attention-deficit/hyperactivity disorder, personality traits, alcohol dependence, panic disorder, schizophrenia, and bipolar disorder. Höpfner et al (15) revealed that changes of hNET level can influence the effect of meta-iodobenzylguanidine on neuroendocrine gastrointestinal tumors $(15-17,32)$. The present study revealed that NET was highly expressed in CRC tissues with metastasis, compared with that found in adjacent normal tissues, and its fold increase was higher than that of patients with non-metastatic CRC. The knockdown of NET resulted in the inhibition of the invasive capability of human colon cancer cells. In addition, E-cadherin expression increased and Notch1 signaling was inhibited upon knockdown of NET in colon cancer cells. These results suggest that high expression of NET in CRC is associated with the metastasis of human colon cancer cells by influencing cell-cell adhesion via the Notch1-E-cadherin pathway.

The activation of the $\beta$-adrenergic system was demonstrated to contribute primarily to stress-associated acceleration of cancer progression (33). NE is the principal chemical messenger employed in central noradrenergic and peripheral sympathetic synapses. Inhibitors of monoamine transmitter reuptake are widely used as antidepressants in clinical practice and are reportedly associated with a decreased risk of CRC (8-10). In addition to their actions against depression, these 
antidepressants also inhibit tumor cell viability $(9,10,34,35)$. The antidepressant fluoxetine was demonstrated to have a direct anti proliferative effect on human colon cancer cells in vitro. Moreover, changes in cell viability, cell cycle, apoptosis and $\mathrm{NF}-\kappa \mathrm{B}$ signaling induced by fluoxetine were also demonstrated. However, the molecular targets associated with this antidepressant were not explored; a series of future experiments will be based on the present findings with the aim of clarifying them and provide evidence for their practice. NET is an important target of antidepressants. Genetic changes or drug interventions are frequently reported to cause several diseases, including cancer. The present study demonstrated high expression of NET to be associated with human colon cancer cell invasion and metastasis. To the best of our knowledge, this is the first study to investigate the target of monoamine transmitter reuptake inhibitors, NET, with respect to its function in human colon cancer cells. However, in vivo studies are required to validate the findings of the present study. NET-targeting short hairpin RNA are currently being constructed, which will be used to knockdown NET in mice for future in vivo studies.

The loss of cell adhesion is a key step in the development of malignancy and metastasis. E-cadherin is a prognostic indicator in metastasis, and its decreased expression was correlated with enhanced metastasis in several malignancies (18-20). In the present study, increased expression of E-cadherin was detected in human colon cancer cells upon the knockdown of NET, which may explain the suppressed invasive capabilities of NET-depleted colon cancer cells Notch signaling, a critical pathway for tissue development, was also shown to be associated with tumorigenesis and tumor metastasis (21). After extracellular activation by Jagged 1, the transmembrane protein Notch is cleaved by $\gamma$-secretase, and the cleaved intracellular domain of Notch activates associated transcription factors to enhance tumor cell metastasis (36). Moreover, Notch signaling regulates E-cadherin expression, and in CRC cells, Notch1-HES1-E-cadherin was demonstrated to promote invasiveness and metastasis (24). The present study also demonstrated the inhibition of Notch1 signaling activity upon knockdown of NET in human colon cancer cells. This result is remarkable in light of a previous report that demonstrated NET function to be involved in noradrenergic cell differentiation; the expression of the Notch signaling inhibitor Numbl was increased, and Notch signaling activity was subsequently inhibited, in both neural crest cells and adult superior cervical ganglion and locus ceruleus in NET-deficient mice (25). NET was highly expressed and associated with metastasis in human CRC tissues, and the depletion of NET inhibited Notch signaling, increased E-cadherin levels and inhibited the invasive capability of human CRC cells. Thus, antidepressants that target and inhibit NET may decrease the risk of human CRC $(8-10,34,35)$. To the best of our knowledge, this is also the first study to describe the molecular mechanisms downstream of NET in human $\mathrm{CRC}$, which requires further investigation.

Overall, the present study revealed a novel function for NET in colon cancer cells and identified its downstream effector molecules, providing valuable information for application in future studies.

\section{Acknowledgements}

The authors would like to thank Professor Hiroyuki Kuwano and Professor Seiji Torii (Graduate School of Medicine, Gunma University, Maebashi, Japan) for their kind assistance; and Mr. Xiaofei Wang, Ms. Lin Yu and Ms. Lei Ni (Key Laboratory of Environment and Genes Related to Diseases, Xi'an Jiaotong University Health Science Center, Xi'an, P.R. China) for their technical support.

\section{Funding}

This work was supported by a grant from the National Natural Science Foundation of China (grant no. 81760510) and the Fundamental Research Funds of Xi'an Jiaotong University (grant no. xjj2016076).

\section{Availability of data and materials}

The datasets used and/or analyzed during the present study are available from the corresponding author upon reasonable request.

\section{Authors' contributions}

$\mathrm{HZ}, \mathrm{JM}, \mathrm{KT}, \mathrm{XZ}, \mathrm{XJ}$ and $\mathrm{NH}$ performed the majority of the experiments and wrote the manuscript. $\mathrm{CH}, \mathrm{NH}$ and $\mathrm{JH}$ supported the design and interpretation of this study. FL, WX and JZ performed the statistical analysis. All authors read and approved the final version of the manuscript.

\section{Ethics approval and consent to participate}

The present study was approved by the Medical Ethics Committee of Xi'an Jiaotong University (Shaanxi, China). All participants provided written informed consent. Personal information for the samples involved in the study was anonymized.

\section{Patient consent for publication}

Not applicable.

\section{Competing interests}

The authors declare that they have no competing interests.

\section{References}

1. Torre LA, Bray F, Siegel RL, Ferlay J, Lortet-Tieulent J and Jemal A: Global cancer statistics, 2012. CA Cancer J Clin 65: 87-108, 2015.

2. Sung JJ, Lau JY, Goh KL and Leung WK; Asia Pacific Working Group on Colorectal Cancer: Increasing incidence of colorectal cancer in asia: Implications for screening. Lancet Oncol 6: 871-876, 2005.

3. Chen W, Zheng R, Baade PD, Zhang S, Zeng H, Bray F, Jemal A, $\mathrm{Yu}$ XQ and He J: Cancer statistics in China, 2015. CA Cancer J Clin 66: 115-132, 2016.

4. Vatandoust S, Price TJ and Karapetis CS: Colorectal cancer: Metastases to a single organ. World J Gastroenterol 21: 11767-11776, 2015.

5. Lutgendorf SK, Sood AK and Antoni MH: Host factors and cancer progression: Biobehavioral signaling pathways and interventions. J Clin Oncol 28: 4094-4099, 2010. 
6. Worster B and Holmes S: The preoperative experience of patients undergoing surgery for colorectal cancer: A phenomenological study. Eur J Oncol Nurs 12: 418-424, 2008.

7. Alberts D, Lluria-Prevatt M, Kha S, Weihs K (eds): Supportive Cancer Care. 1st ed. Springer International Publishing, Switzerland, pp45-76, 2016

8. Chubak J, Boudreau DM, Rulyak SJ and Mandelson MT: Colorectal cancer risk in relation to antidepressant medication use. Int J Cancer 128: 227-232, 2011.

9. Koh SJ, Kim JM, Kim LK, Kim N, Jung HC, Song IS and Kim JS: Fluoxetine inhibits NF- $\kappa$ B signaling in intestinal epithelial cells and ameliorates experimental colitis and colitis-associated colon cancer in mice. Am J Physiol Gastrointest Liver Physiol 301: G9-G19, 2011.

10. Kannen V, Garcia SB, Silva WA Jr, Dasser M, Mönch R, Alho EJ, Heinsen H, Scholz CJ, Friedrich M, Heinze KG, et al: Oncostatic effects of fluoxetine in experimental colon cancer models. Cell Signal 27: 1781-1788, 2015.

11. Kristensen AS, Andersen J, Jørgensen TN, Sørensen L, Eriksen J, Loland CJ, Strømgaard K and Gether U: SLC6 neurotransmitter transporters: Structure, function and regulation. Pharmacol Rev 63: 585-640, 2011.

12. Mandela P and Ordway GA: The norepinephrine transporter and its regulation. J Neurochem 97: 310-313, 2006.

13. Pramod AB, Foster J, Carvelli L and Henry LK: SLC6 transporters: Structure, function, regulation, disease association and therapeutics. Mol Aspects Med 34: 197-219, 2013.

14. Zahniser NR and Doolen S: Chronic and acute regulation of $\mathrm{Na}^{+} / \mathrm{Cl}^{-}$dependent neurotransmitter transporters: Drugs, substrates, presynaptic receptors, and signaling systems. Pharmacol Ther 92: 21-55, 2001.

15. Höpfner M, Sutter AP, Huether A, Ahnert-Hilger G and Scherübl H: A novel approach in the treatment of neuroendocrine gastrointestinal tumors: Additive antiproliferative effects of interferon-gamma and meta-iodobenzylguanidine. BMC Cancer 4: 23, 2004.

16. Hahn MK and Blakely RD: The functional impact of SLC6 transporter genetic variation. Annu Rev Pharmacol Toxicol 47: 401-441, 2007.

17. Matthay KK, George RE and Yu AL: Promising therapeutic targets in neuroblastoma. Clin Cancer Res 18: 2740-2753, 2012.

18. Cavallaro $U$ and Christofori G: Cell adhesion and signaling by cadherins and Ig-CAMs in cancer. Nat Rev Cancer 4: 118-132, 2004

19. Weiss JV, Klein-Scory S, Kübler S, Reinacher-Schick A, Stricker I, Schmiegel W and Schwarte-Waldhoff I: Soluble E-cadherin as a serum biomarker candidate: Elevated levels in patients with late-stage colorectal carcinoma and FAP. Int J Cancer 128: 1384-1392, 2011.

20. Okugawa $Y$, Toiyama $Y$, Inoue $Y$, Iwata $T$, Fujikawa $H$, Saigusa $S$, Konishi N, Tanaka K, Uchida K and Kusunoki M: Clinical significance of serum soluble E-cadherin in colorectal carcinoma. J Surg Res 175: e67-e73, 2012.

21. Hu YY, Zheng MH, Zhang R, Liang YM and Han H: Notch signaling pathway and cancer metastasis. Adv Exp Med Biol 727 186-198, 2012

22. Chen J, Imanaka N, Chen J and Griffin JD: Hypoxia potentiates notch signaling in breast cancer leading to decreased E-cadherin expression and increased cell migration and invasion. Br J Cancer 102: 351-360, 2009.
23. Wang XQ, Zhang W, Lui EL, Zhu Y, Lu P, Yu X, Sun J, Yang S, Poon RT and Fan ST: Notch1-Snail1-E-cadherin pathway in metastastic hepatocellular carcinoma. Int J Cancer 131: E163-E172, 2012

24. Yuan R, Ke J, Sun L, He Z, Zou Y, He X, Chen Y, Wu X, Cai Z, Wang L, et al: HES1 promotes metastasis and predicts poor survival in patients with colorectal cancer. Clin Exp Metastasis 32: 169-179, 2015.

25. Hu YF, Caron MG and Sieber-Blum M: Norepinephrine transport-mediated gene expression in noradrenergic neurogenesis. BMC Genomics 10: 151, 2009.

26. Cancer Genome Atlas Network: Comprehensive molecular characterization of human colon and rectal cancer. Nature 487: 330-337, 2012.

27. Zhang MY, Liu XX, Li H, Li R, Liu X and Qu YQ: Elevates mRNA levels of AURKA, CDC20 and TPX2 are associated with poor prognosis of smoking related lung adenocarcinoma using bioinformatics analysis. Int J Med Sci 15: 1676-1685, 2018.

28. Hou N, Zhang X, Zhao LY, Zhao XG, Li ZF, Song TS and Huang C: A novel chronic stress-induced shift in the Th1 to Th2 response promotes colon cancer growth. Biochem Biophys Res Commun 439: 471-476, 2013.

29. Zhou X, Zhao F, Wang ZN, Song YX, Chang H, Chiang Y and $\mathrm{Xu}$ HM: Altered expression of miR-152 and miR-148a in ovarian cancer is related to cell proliferation. Oncol Rep 27: 447-454, 2012.

30. Ouyang W, Ren L, Liu G, Chi X and Wei H: LncRNA MIR4435-2HG predicts poor prognosis in patients with colorectal cancer. Peer J 7: e6683, 2019.

31. Tania M, Khan MA and Fu J: Epithelial to mesenchymal transition inducing transcription factors and metastatic cancer. Tumor Biol 35: 7335-7342, 2014.

32. Pandit-Taskar N and Modak S: Norepinephrine transporter as a target for imaging and therapy. J Nucl Med 58 (Suppl 2): 39S-53S, 2017.

33. Tang J, Li Z, Lu L and Cho CH: $\beta$-Adrenergic system, a backstage manipulator regulating tumour progression and drug target in cancer therapy. Semin Cancer Biol 23: 533-542, 2013.

34. Kannen V, Hintzche H, Zanette DL, Silva WA Jr, Garcia SB, Waaga-Gasser AM and Stopper H: Antiproliferative effects of fluoxetine on colon cancer cells and in a colonic carcinogen mouse model. PLoS One 7: e50043, 2012.

35. Stopper H, Garcia SB, Waaga-Gasser AM and Kannen V: Antidepressant fluoxetine and its potential against colon tumors. World J Gastrointest Oncol 6: 11-21, 2014.

36. Leong KG, Niessen K, Kulic I, Raouf A, Eaves C, Pollet I and Karsan A: Jagged1-mediated notch activation induces epithelialto-mesenchymal transition through Slug-induced repression of E-cadherin. J Exp Med 204: 2935-2948, 2007.

This work is licensed under a Creative Commons Attribution-NonCommercial-NoDerivatives 4.0 International (CC BY-NC-ND 4.0) License. 